TITLE:

\title{
Evaluation of grouting in tunnel lining using impact-echo
}

$\operatorname{AUTHOR}(S)$ :

AGGELIS, D.G.; SHIOTANI, T.; KASAI, K.

CITATION:

AGGELIS, D.G.... [et al]. Evaluation of grouting in tunnel lining using impact-echo. Tunnelling and Underground Space Technology 2008, 23(6): 629-637

ISSUE DATE:

2008-11

URL:

http://hdl.handle.net/2433/93470

\section{RIGHT:}

Copyright (c) 2007 Elsevier; This is not the published version. Please cite only the published version.; この論文は出版社版でありません。引用の 際には出版社版をご確認ご利用ください。 


\title{
Evaluation of grouting in tunnel lining using impact-echo
}

\author{
D. G. Aggelis ${ }^{\mathrm{a}}$, T. Shiotani*b ${ }^{*}$, K. Kasai ${ }^{\mathrm{c}}$ \\ ${ }^{a}$ Research Institute of Technology,Tobishima Corporation \\ 5472 Kimagase, Noda, Chiba, 270-0222, Japan \\ ${ }^{b}$ Department of Urban Management, Graduate School of Engineering, Kyoto University, C1-2-236, \\ Kyoto-Daigaku-Katsura, Nishikyo-Ku Kyoto 615-8540, Japan, \\ ${ }^{c}$ Technology Management Department, Civil Engineering Headquarters, Tobishima Corporation \\ 2-Banchi, Sanbancho, Chiyoda-ku, Tokyo, 102-8332, Japan
}

\begin{abstract}
The Shield Tunneling Method is commonly used for tunnel excavation. After the excavation, curved reinforced concrete members are used to support the surrounding ground/rock and seal the tunnel. Grouting is performed to provide adhesion between the concrete members and the ground. The assessment of the grouting quality by pull-out tests and core sampling is destructive, time-consuming and expensive. In the present case, impact-echo was applied as a means of non destructive and time-effective evaluation. Excitation was conducted by an impact hammer on the surface of several concrete plates and the reflection was acquired by a low frequency accelerometer. Combined use of time domain characteristics, spectral content and wavelet transform reveals the effectiveness of grouting and indicate that impact echo is valuable for quick and reliable assessment of grouting in such cases.
\end{abstract}

Keywords: Impact-echo, Non-destructive inspection, grouting,

\footnotetext{
* Corresponding author. Tel:+81-4-7198-7572, Fax:+81-4-7198-7586. E-mail address: shiotani@df7.so-net.ne.jp
} 


\section{Introduction}

The Shield Tunneling Method is a common method for excavation of small diameter tunnels. Immediately after the excavation by means of tunnel boring machine, the surrounding material must be supported and the cross-section of the tunnel secured (Darrag, 1999). For this aim, prefabricated curved concrete segments are assembled, and injection of grout is conducted to fill the void between the segment and the excavated surface. This way, the surrounding rock mass is supported and the stresses are conveyed to the reinforced concrete segments. Therefore, it is important that the lining is fully attached and no voids exist between the surrounding ground and the concrete segment. Although the conditions of the injection are adequately controlled, there is no confirmation that the grout material has filled all the available empty volume between the concrete segment and the ground. Efforts to estimate the grouted volume are based on the geometry, the permeability of the rock and the applied pressure but in any case cannot supply reliable information (Brantberger, 2000). The assessment of the efficacy of grouting is not trivial (Suda et al., 2004). One way is the core sampling, which of course is destructive and not representative of large area.

The tunnel of this study is $2.3 \mathrm{~km}$ long, with diameter of $5 \mathrm{~m}$. It was built for telecommunication cables and sewage pipes. The location is in the central area of Japan, and the ground consisted mainly of low quality mudstone. After placement of the concrete members and the injection of grout, the quality of the filling should be assessed with a non destructive technique; therefore, impact-echo was applied.

Impact-echo generally employs the spectral analysis of the response of the member after a low frequency excitation. Reflections are recorded according to the material's internal conditions, 
and their frequency can reveal information about the thickness of the member, the depth of defects, as well as, material properties (Sansalone and Streett, 1997, Ohtsu and Watanabe, 2002). Specifically for the tunnel of this case study, although information was collected from the spectral content, more reliable results were obtained by characteristics of the time domain waveform, combination of which is used to distinguish between the fully and partially grouted cases.

\section{Materials and Experimental method}

In order to make specific calculations about the expected frequency peaks after the test, except from the geometry of the structure, the material properties are essential. Cores from the surrounding mudstone were sampled at the site and specimens of grouting material were prepared and tested in the laboratory to obtain their pulse velocity. The pulse velocity measurements were conducted with acoustic emission resonant R6 sensors of Physical Acoustics Corp. (PAC), the pulse generator was a PAC HV-101 and the data acquisition system was a PAC Mistras 2001. The pulse velocities and densities of the materials are depicted in Table 1.

The velocity of concrete is close to the highest possible limit for cementitious material (Naik and Malhotra, 1991) due to the high quality of manufacturing and high integrity conditions (no accumulated deterioration) of the metal-reinforced segment. The design strength of concrete was $42.5 \mathrm{MPa}$ and according to the measured pulse velocity the modulus of elasticity is over $60 \mathrm{GPa}$. The velocity of mudstone indicates low mechanical properties, (elasticity modulus of the order of $4 \mathrm{GPa}$ ). The properties of this material could not be controlled (since it was the 
surrounding material at the site) and the velocity was measured to facilitate the analysis of the results. Concerning grouting material; leaving the least possible number of voids was the main target of grouting. Therefore, the grouting agent should be as liquid and easy-to-flow as possible and the engineers at site selected a high water to solid ratio to ensure this condition. The grouting material consisted mainly of bentonite, a smaller proportion of assistant cementitious material, accelerator agent and water. The proportions were $200 \mathrm{~kg} / 50 \mathrm{~kg} / 65 \mathrm{lt} /$ 850 lt respectively for $1 \mathrm{~m}^{3}$ of the grouting material. This led to low pulse velocity, similar to the mudstone (as seen in Table 1) and calculated elasticity modulus around $3 \mathrm{GPa}$. In case lower water content was used, higher mechanical properties and velocity would be acquired but possibly more voids would be left after the end of the process; thus it was a compromise between strength and flowability, mainly based on the experience of the engineers at site.

As seen in Table 1, the properties of mudstone were slightly higher than the grout ones. This resulted in similar acoustic impedances for the two materials, meaning that at the interface between the two materials, a strong reflection is not expected, as will be discussed later. According to the manufacturer, the gel time of grout is 6-12 s, the flow time $8-11 \mathrm{~s}$ and the set time 25-35 min. In proportion $1 / 4$ with water, it acquires compressive strength of $0.06 \mathrm{MPa}$ in 1 hr and $1.5 \mathrm{MPa}$ at 28 days. The mudstone at the site had strength of $4 \mathrm{MPa}$, measured in laboratory on retrieved cylindrical cores of length $100 \mathrm{~mm}$ and diameter $50 \mathrm{~mm}$.

As to the main measurements at the tunnel site for the evaluation of grouting, the excitation was conducted by an impact hammer, as seen in Fig. 1. The impact hammer diameter was 25 $\mathrm{mm}$ although smaller diameters were tested, down to $3 \mathrm{~mm}$. This diameter was selected since it resulted in high energy and clearer signal. Theoretically, the maximum frequency excited by a 
steel ball of diameter $25 \mathrm{~mm}$, is $11.5 \mathrm{kHz}$ (Sansalone and Streett, 1997). Hammering was repeatedly conducted for $10 \mathrm{~s}$ resulting in acquisition of twenty individual signals. However, the signal was strong enough and stacking did not reveal difference in spectral content from the individual waveforms. The used accelerometers were Fujiceramics SAF51 with flat response up to $30 \mathrm{kHz}$ and the signal was acquired by a TEAC GX-1 system. The sampling rate was set to the maximum available value of $200 \mathrm{kHz}$.

Fig. 2 shows the cross section of the tunnel. The reinforced concrete segments, used to support the mudstone, had a thickness of $225 \mathrm{~mm}$. They can be considered arches of $68.5^{\circ}$ of a circle with external diameter of $5450 \mathrm{~mm}$ (see Fig. 2). Five full scale segments and a smaller one were used to seal the cross-section, consisting a "ring". To seal the whole length of the tunnel, several thousands of consecutive rings were placed, while the width of the segment (and the ring) was $1200 \mathrm{~mm}$.

The grouting was performed at each one out of two consecutive rings from a hole in the center of a top segment. Using the excavation diameter $(5.6 \mathrm{~m})$ the estimated empty volume between the concrete segment and mudstone, as well as the sufficient quantity of grouting material was calculated. Due to uncertainties related to this estimation the actually injected grout volume exceeded this value by $30 \%$, while the existence of grouting material was visually observed from the next holes. The maximum injection pressure was $35 \mathrm{MPa}$. However, although the pressure of the injection and the volume of injection material can be adequately controlled, there is no confirmation that the grout material has filled all the available empty volume between the concrete segment and the ground. 
The pulse velocity in a concrete segment was determined by a through-the-thickness measurement, resulting in $5000 \mathrm{~m} / \mathrm{s}$, as stated earlier. Considering the major frequency and this pulse velocity, the wavelength in concrete is calculated to $500 \mathrm{~mm}$, being much longer than the diameter of the reinforcement bars of 13 and $16 \mathrm{~mm}$ in the segment, and therefore strong interaction is not expected.

\section{Wave propagation background}

Since the material properties were derived, more specific calculations can be conducted, concerning the expected frequencies of the test. After excitation at an impact point, the wave propagates through the thickness of the segment, until it reaches the opposite surface (see Fig. 3(a)). At the interface between the concrete segment and bentonite a part of wave energy is reflected back. This is because the acoustic impedance of stiff concrete is much higher than the one of bentonite (12.5 MRayl and 1.97 MRayl respectively) as seen in Table 1. The reflection coefficient is given by (Sansalone and Carino, 1991):

$R=\frac{Z_{2}-Z_{1}}{Z_{2}+Z_{1}}$

where $Z_{1}$ and $Z_{2}$ are the acoustic impedances of the first and second material (in this case concrete and bentonite respectively).

It is noted that the impedance $\mathrm{Z}$, is the product of density and pulse velocity of a medium (Sansalone and Carino, 1991). 
At the interface of concrete and bentonite, the reflection is quite high, $\mathrm{R}=-0.727$, showing that the reflected wave will have amplitude approximately $73 \%$ of the incident wave (the negative sign shows that the phase of the wave is reversed, but this will not be discussed in this paper). Considering the P-wave velocity of $5000 \mathrm{~m} / \mathrm{s}$ and the thickness of $225 \mathrm{~mm}$, it is easily calculated that the time period necessary for the pulse to travel through the thickness and backwards is $90 \mu \mathrm{s}$, which inversed, corresponds to $11 \mathrm{kHz}$. The curvature of the segments was comparatively small and, alternatively, the following expression can be used (Sansalone and Streett, 1997):

$$
f=\frac{0.96 \cdot C}{2 \cdot T}
$$

where $\mathrm{T}$ is the thickness and $\mathrm{C}$ the pulse velocity.

This leads to a frequency of approximately $10.6 \mathrm{kHz}$. Thus, in any case, a strong peak in the received fast Fourier transform $(\mathrm{FFT})$ is expected above $10 \mathrm{kHz}$.

Besides the energy that is reflected by the concrete - grout interface, there is an amount of energy that enters in grout. This part of energy propagates through the thickness of grout until it reaches the next boundary. In case the grouting is complete, the grouting material will be in direct contact with the mudstone (see Fig. 3(a)). As mentioned earlier, the impedances of these two materials are very close and therefore, the reflection coefficient will be very low $(\mathrm{R}=0.09)$. In order a reflection to be clear, a coefficient of more than 0.24 is necessary (Sansalone and Streett, 1997). Therefore, almost all the energy will propagate into the mudstone away from the 
receiver. This means that only one major frequency peak at approximately $11 \mathrm{kHz}$ is expected for the fully grouted case.

If the grouting is not complete, a void will be present between bentonite and mudstone, as depicted in Fig. 3(b). Since air has negligible impedance, the reflection will be strong $(\mathrm{R}=-1)$. The resulting frequency in the spectrum will depend on the propagation time through the bentonite (grouting) layer and therefore, its thickness. For example, for bentonite layer thickness of $70 \mathrm{~mm}$, the propagation time would be approximately $85 \mu$ s (considering its pulse velocity of $1650 \mathrm{~m} / \mathrm{s}$ ). In addition to the $90 \mu \mathrm{s}$ through concrete, this leads to an expected frequency peak at $5.5 \mathrm{kHz}$. Therefore, a second lower frequency peak should arise in the spectrum, indicating the void between the grout and the surrounding mudstone.

\section{Results}

Before applying the method to rings throughout the actual structure, measurements were conducted under controlled conditions on several positions near the entrance of the tunnel. There are different cases simulated namely complete and partial grouting, as can be seen in Fig. 4(a). Fig. 4(b) shows the detail of the partial case with the grout layer of about $70 \mathrm{~mm}$.

\subsection{Spectral content}

Fig. 5(a) shows typical fast Fourier transforms (FFT) of totally filled and partially filled cases. The FFT of fully filled, exhibits a single peak just above $10 \mathrm{kHz}$, corresponding to the thickness of the segment, as explained earlier. In the case of partial filling, except the high frequency, another clear peak is seen at around $6 \mathrm{kHz}$. However, not all cases could be 
distinguished easily. In Fig. 5(b) two other indicative cases are presented. Concerning the fully filled position, a small frequency peak at $5 \mathrm{kHz}$ can be observed. This peak could cast doubt if the filling was not visually confirmed. On the other hand, the case of partial filling in Fig. 5(b) exhibits again a similar small peak at low frequencies. Therefore, the existence of this peak should not be the unique criterion for characterization, since it can be observed in both fully and partially grouted conditions. It could be the result of the reflection due to a void or the reflection between grout and mudstone in the fully filled case (even though theoretically it is expected to be weak according to the acoustic impedance values, as mentioned above).

A powerful way to visualize the response is the use of wavelets, which can offer valuable information. Wavelet transformation is a means of conducting frequency analysis in a localized part of a signal. Therefore, it is possible to identify the time within a waveform when each frequency component is active (as opposed to the Fourier transform that gives information about the frequency content of the whole signal as an entity). The Wavelet Transform (WT) works by breaking the signal into shifted and scaled versions of the original wavelet (fast decaying mathematical function) (Misiti et al. 1996). In order to handle suitably any signal, different original ("mother") wavelet functions can be used. In the specific case, the software used by the authors (AGU-Vallen Wavelet) employs the "Gabor" Wavelet, which has proven very useful for analysis of ultrasonic and acoustic emission signals (Hamstad, 2005). The results show that it is adequate for the analysis, since it helps the identification of different cases, while possibly a number of other mother wavelets would produce similar results. In Fig. 6(a) and (b) one can see the wavelet transform of waveforms corresponding to fully filled condition. The major part of energy is concentrated before $200 \mu$ s and almost no energy is depicted after $500 \mu \mathrm{s}$. For the case of partial grouting though (see Fig. 6(c) and (d)), the energy 
is noticeable until about $700 \mu \mathrm{s}$. Additionally, a distinct energy component at $5 \mathrm{kHz}$ is more evident, surviving up to approximately $400 \mu$ s (especially in Fig. 6(c)), corresponding to the lower peak of the FFT discussed earlier. Visual observation can serve the purpose of rough characterization; however, there is not a decisive difference between the filled and not filled cases, since the low frequency component can be seen in both healthy and partial conditions (see Fig. 6(b) and (d)). The visual inspection of wavelet transformation can distinguish between some extreme cases, but it is not adequate for reliable quantification purposes. Therefore, other features were studied in order to lead to a more robust characterization scheme. These features come from the time domain and, although they are not commonly examined in impact-echo testing, they led to more powerful characterization than frequency features in this case.

\subsection{Time domain information}

Fig. 7(a) shows typical waveforms obtained for fully and partially filled positions. For the case of full grouting, the waveform consists of one single burst that decays smoothly. Almost all the energy arrives before the $500 \mu$ s, while no threshold crossings (see horizontal dash-dot line) are observed afterwards (except a number of small high-frequency cycles which are better depicted in Fig. 7(b) and will be discussed later). This is reasonable since the reflection from concretegrout interface is the only strong one, and the energy propagating through the grout will never be reflected back, due to the similarity of grout and mudstone, as explained earlier. For the case of partial filling though (see again Fig. 7(a)), the decay is not smooth, presumably due to the arrival of the late reflection expected at around $170 \mu \mathrm{s}$. Additionally, even after $500 \mu$ s, some low frequency oscillations are observed. 
In order to quantify this trend, the number of threshold crossings or simply "counts" in acoustic emission terminology (Shiotani et al. 2003) is examined. It is reasonable that the threshold voltage is of paramount importance for such a descriptor. In Fig. 8(a), one can see the number of counts for threshold equal to $1 \%$ of the maximum voltage for each waveform. The positions 1 to 5 correspond to full grouting, while 6 to 11 to partial, as simulated in the entrance of the tunnel (see Fig. 4(a)). It is seen that most of the fully grouted positions exhibit lower counts than partial; however, this does not hold for all the cases since position 5, although considered fully grouted, exhibits the highest number of crossings. If the threshold is set to $10 \%$, only larger oscillations are counted and the results are depicted in Fig. 8(b). It is obvious that this threshold provides better discrimination, although there is some overlap concerning the number of 6 counts that is common for filled and unfilled positions. The results are improved if one considers the counts of the later part of the waveform. As seen in Fig. 7(a), the filled case exhibits significant energy up to about $500 \mu \mathrm{s}$, while for the partial case, the energy expands up to $800 \mu \mathrm{s}$. Therefore, in Fig. 8(c) one can see the counts after $500 \mu \mathrm{s}$. In this case characterization is much easier, since there is no overlap; the fully filled cases exhibit 0 or 1 count, while the partial exhibit at least 3 or more.

As stated earlier, and seen in Fig. 7(b), the waveforms of fully grouted positions exhibit numerous low-voltage, high-frequency oscillations after the initial burst. These can be examined by a suitably low threshold of $0.1 \%$. This seems to be another also powerful descriptor, since the full cases exhibit numerous low, high-frequency counts compared to the partial (see Fig. 8(d)). 
It should be mentioned that understanding of wave propagation is not always easy in a comple $\mathrm{x}$ structure like the one at hand. In this case the origin of the low-energy, high-frequency oscillations seen at the last part of the waveform cannot be confirmed. Presumably, it is connected with interaction of the wave with the metal reinforcement of the segments even though their diameter is smaller than the major wavelength. In any case the certain fact is that they are observed in great numbers only in the fully-filled cases. Therefore, they could be used as stand-alone features or in combination with others to lead to reliable characterization, when individual features are not powerful enough.

Although the number of data is limited and, in this case, only one of the features could result in successful characterization, the combination of different features in a multidimensional analysis provides highlighted results.

In Fig. 9, the eleven reference cases are depicted in a three dimensional graph with axes: total counts with threshold $10 \%$ of the maximum, counts after $500 \mu$ s with the same threshold and counts after $500 \mu \mathrm{s}$ with the low threshold of $0.1 \%$. It is obvious that the results form two clearly separated clusters. This could be the first step of a pattern recognition approach if the problem of characterization is difficult and one single descriptor is not powerful enough.

\subsection{Other time domain features}

Another descriptor that seems characteristic is the waveform central time (or the centroid of the rectified waveform), which has been studied in other cases (Aggelis and Philippidis, 2004). As can be seen in the examples of Fig. 10(a), the filled positions exhibit a single burst followed by 
small cycles. For the partial cases though, the burst lasts longer, while more, strong oscillations follow, translating the centroid of the signal to later times. For any of the intact positions, the central time is below $500 \mu \mathrm{s}$, while for all the partially filled cases it is higher than $550 \mu \mathrm{s}$, as can be seen in Fig. 10(b).

An additional feature that could be used to quantify the later arrivals is the accumulated amplitude of the waveform points. This is calculated by addition of the absolute amplitude of each waveform point to the previous (Kruger, 2005, Shiotani and Aggelis, 2007). In Fig. 11(a) one can see the accumulated amplitude curve of the waveforms for the different reference positions. For all the curves of fully-filled positions, more than $75 \%$ of the total energy is received before $500 \mu \mathrm{s}$. On the contrary, for the cases of partial filling, at the time of $500 \mu \mathrm{s}$, the energy is less than $70 \%$ of the total (see Fig. 11(a)), since, as stated earlier, some energy components arrive later. This parameter is depicted in Fig. 11(b) for different positions and can also be used to characterize the filling since no overlap between the full and partial cases is observed.

\section{Further considerations}

The main wave propagation issues and features that can help the characterization were mentioned in the previous section. After selection of the above features, measurements on ten rings were conducted. For each ring, eight positions were tested, as depicted in Fig. 2. The results were satisfactory for almost the sum of the points. Troublesome results were found only for position \#2 in specific rings. This is reasonable since the fresh grout tends to fill the lower parts due to gravity, and therefore, position \#5 exhibited the best behavior (low number of late counts and central time, fast increase of accumulated energy). Since top positions exhibit 
higher possibility for troublesome grouting, it can be suggested that two or three points on the roof of each ring can be targeted, and if time allows, measurements can be conducted in a denser pattern and for more rings.

The above descriptors can distinguish between completely and not completely grouted spots. The determination of the bentonite layer thickness in partial filling is a much more difficult task. Estimation could be done, based on the frequency of the low peak of the spectrum. For example, frequency of $4 \mathrm{kHz}$ should correspond to grouting layer thickness of $120 \mathrm{~mm}$, while frequency of $7 \mathrm{kHz}$ would indicate grouting of $40 \mathrm{~mm}$. However, characterization based on the low frequency peak is not reliable, as stated earlier. In any case, the crucial question concerns the adhesion between bentonite and the surrounding ground. If the grouting is not full, no adhesion can be developed, since there is no contact between the materials. Therefore, even if the empty pocket is just a few millimeters or several centimeters the same lack of adhesion will be the result and more accurate characterization would not offer comparatively large benefit.

A complication in the interpretation of data would arise if instead of a possible void between bentonite and mudstone, the cavity was filled with water. This could happen in certain locations where there is underground water flow. Considering the density and sound velocity in water $\left(1000 \mathrm{~kg} / \mathrm{m}^{3}\right.$ and $1500 \mathrm{~m} / \mathrm{s}$ respectively), its acoustic impedance is of the same order with bentonite, as well as mudstone. Therefore, a reflection would not be observed and this case could be mistaken for a fully grouted case.

Considering the thickness of the segment, higher frequency excitation is supposed to reveal more accurate information (Sansalone and Streett, 1997). However, application of smaller 
diameters of impact hammer, led to reduced energy and therefore, analysis of obtained features did not result in reliable characterization.

It is also mentioned that the above presented features served well the purpose of the specific monitoring. It is suggested that these features could be used (individually or combined if necessary) to characterize similar cases. However, the calculated or expected values for each descriptor (e.g. frequency peak or number of threshold crossings) which were used to distinguish complete from troublesome grouting, should be modified according to the geometry and the material properties in a case by case basis after reference measurements for any different application.

\section{Conclusions}

In the present paper, the impact-echo method is used to investigate the filling between the tunnel lining and the surrounding mudstone. Troublesome grouting is indicated by a strong reflection from the existent void which leads to a distinctive peak in the Fourier spectrum. Wavelet transformation of the received signal is a powerful way to visualize the energy content in relation to time and its frequency content. However, time domain characteristics, like the number of threshold crossings, or the delay of major energy arrivals seem more powerful to distinguish between fully or not fully grouted cases. It is concluded that the impact-echo technique can be used as a non destructive evaluation method since it is time and cost effective and has the ability to examine unlimited number of positions, as opposed to traditional core drilling. 


\section{References}

Aggelis, D.G., Philippidis, T.P. 2004. Ultrasonic wave dispersion and attenuation in fresh mortar. NDT \& E Int 37 (8), 617-631.

AGU-Vallen Wavelet, R2005.1121, www.vallen.de

Brantberger, M., Stille, H., Eriksson, M. 2000. Controlling grout spreading in tunnel grouting - analyses and developments of the GIN-method. Tunnelling and Underground Space Technology 15 (4), 343-352

Darrag, A. A., 1999. Ground stabilization for tunnel construction in mixed-face conditions. Tunnelling and Underground Space Technology 14 (3), 319-326.

Hamstad M. A., 2005. An Illustrated Overview of the Use and Value of a Wavelet Transformation to Acoustic Emission Technology, AGU-Vallen Wavelet R2005.1121, User's Guide.

Kruger, M., 2005. Scanning impact-echo techniques for crack depth determination. OttoGraf-Journal 16, 245-257.

Misiti M., Misiti Y., Oppenheim G., Poggi J., 1996. Matlab Wavelet Toolbox User's Guide.

Naik T. R., Malhotra V. M., 1991. The Ultrasonic Pulse Velocity Method, in CRC Handbook on Nondestructive Testing of Concrete, V.M. Malhotra, N. J. Carino eds. CRC Press, Florida, 169-188.

Ohtsu, M., Watanabe, T., 2002. Stack imaging of spectral amplitudes based on impactecho for flaw detection. NDT\&E Int 35 (3), 189-196.

Sansalone, M. J., Carino, N., 1991. Stress Wave Propagation Methods, in CRC Handbook on Nondestructive Testing of Concrete, V.M. Malhotra, N. J. Carino eds. CRC Press, Florida, 1991 (p. 278). 
Sansalone, M. J., Streett, W. B., 1997. Impact-echo nondestructive evaluation of concrete and masonry. Bullbrier Press, Ithaca, NY.

Shiotani, T., Aggelis, D.G., 2007. Determination of Surface Crack Depth and Repair Effectiveness using Rayleigh Waves. In: Carpinteri A, Gambarova P, Ferro G, Plizzari G, (eds) Fracture Mechanics of Concrete and Concrete Structures - Design, Assessment and Retrofitting of RC Structures, Taylor \& Francis, London, UK, 1011-1018.

Shiotani, T., Bisschop, J., Van Mier, J.G.M., 2003. Temporal and spatial development of drying shrinkage cracking in cement-based materials. Engineering Fracture Mechanics 70 (12), 1509-1525.

Suda, T., Tabata, A., Kawakami, J., Suzuki, T., 2004. Development of an impact sound diagnosis system for tunnel concrete lining. Tunnelling and Underground Space Technology 19, 328-329. 
Table 1. Material properties

\begin{tabular}{|c|c|c|c|}
\hline & Concrete & Grouting & Mudstone \\
& segment & material & \\
\hline C, wave speed $(\mathrm{m} / \mathrm{s})$ & 5000 & 1631 & 1823 \\
\hline$\rho$, density $\left(\mathrm{kg} / \mathrm{m}^{3}\right)$ & 2500 & 1205 & 1297 \\
\hline Z (acoustic & 12.5 & 1.97 & 2.36 \\
impedance, MRayl) & & & \\
\hline
\end{tabular}


Figure captions

Fig. 1 Excitation using an impact hammer of $25 \mathrm{~mm}$ near the accelerometer.

Fig. 2 Cross-section of the concrete ring, consisting of six segments. (The dimensions are in $\mathrm{mm})$.

Fig. 3 Reflections according to the internal geometry (a) fully filled case, (b) partially filled case.

Fig. 4 (a) Different filling cases simulated at the entrance of the tunnel, (b) detail of partial filling case.

Fig. 5 FFT of different filling cases. (a) distinguishable cases, (b) not distinguishable cases.

Fig. 6 Wavelet transforms of responses from: (a) and (b) fully filled, (c) and (d) partially filled position.

Fig. 7 (a) Waveforms from different filling cases with threshold $10 \%$ of the maximum, (b) the waveforms magnified after $500 \mu \mathrm{s}$, with threshold $0.1 \%$.

Fig. 8 Number of (a) counts for threshold $1 \%$ of maximum voltage, (b) counts for threshold $10 \%$ of maximum voltage, (c) counts after $500 \mu$ s for threshold $10 \%$, (d) counts after $500 \mu$ s for threshold $0.1 \%$ (positions $1-5$ correspond to full filling and 6-11 to partial filling).

Fig. 9 Three dimensional representation of different clusters.

Fig. 10 (a) Rectified waveforms for different grouting cases, (b) central waveform time for different testing points (positions 1-5 correspond to full filling and 6-11 to partial filling).

Fig. 11 (a) Normalized cumulative energy for different testing points, (b) Normalized cumulative energy at the time of $500 \mu$ s (positions 1-5 correspond to full filling and 6-11 to partial filling). 


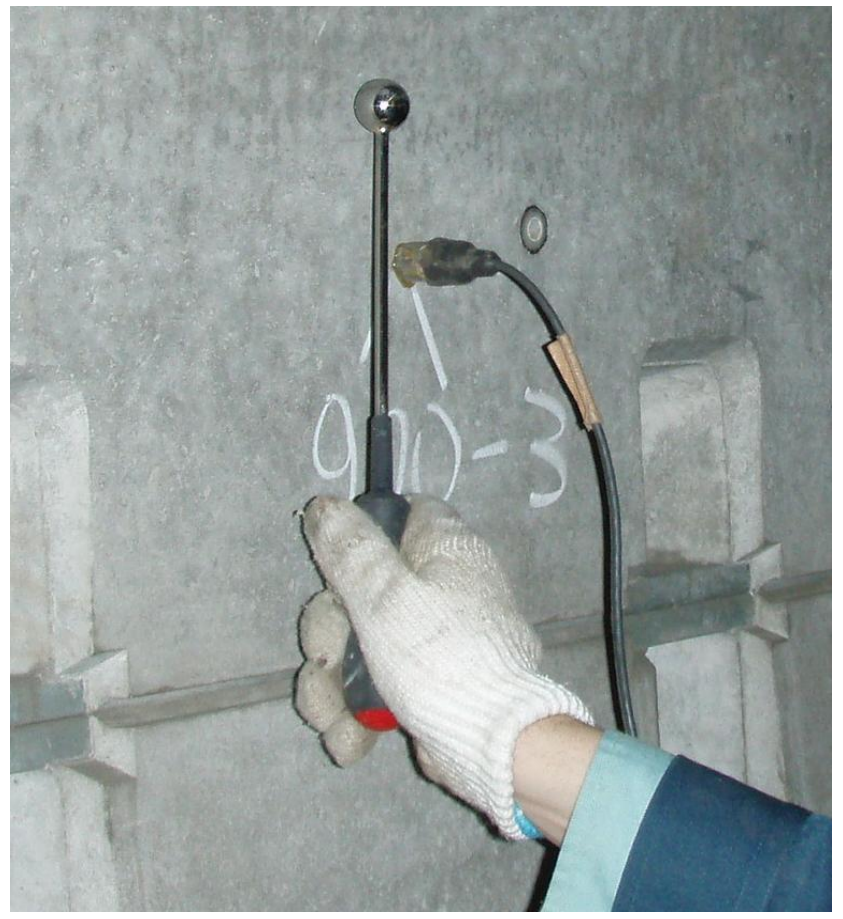

Fig. 1 Excitation using impact hammer of $25 \mathrm{~mm}$ near the accelerometer. 


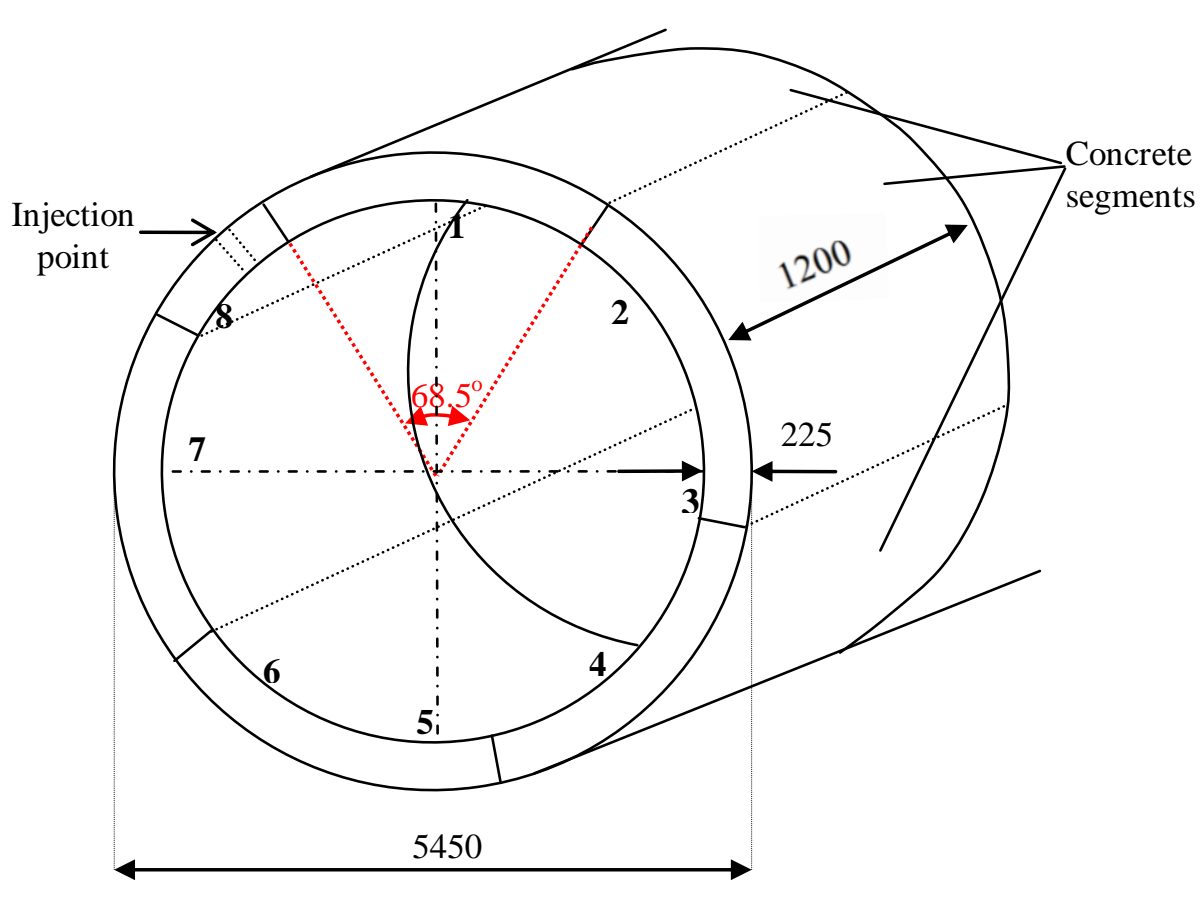

Fig. 2 Cross-section of the concrete ring, consisting of six segments. (The dimensions are in $\mathrm{mm})$. 


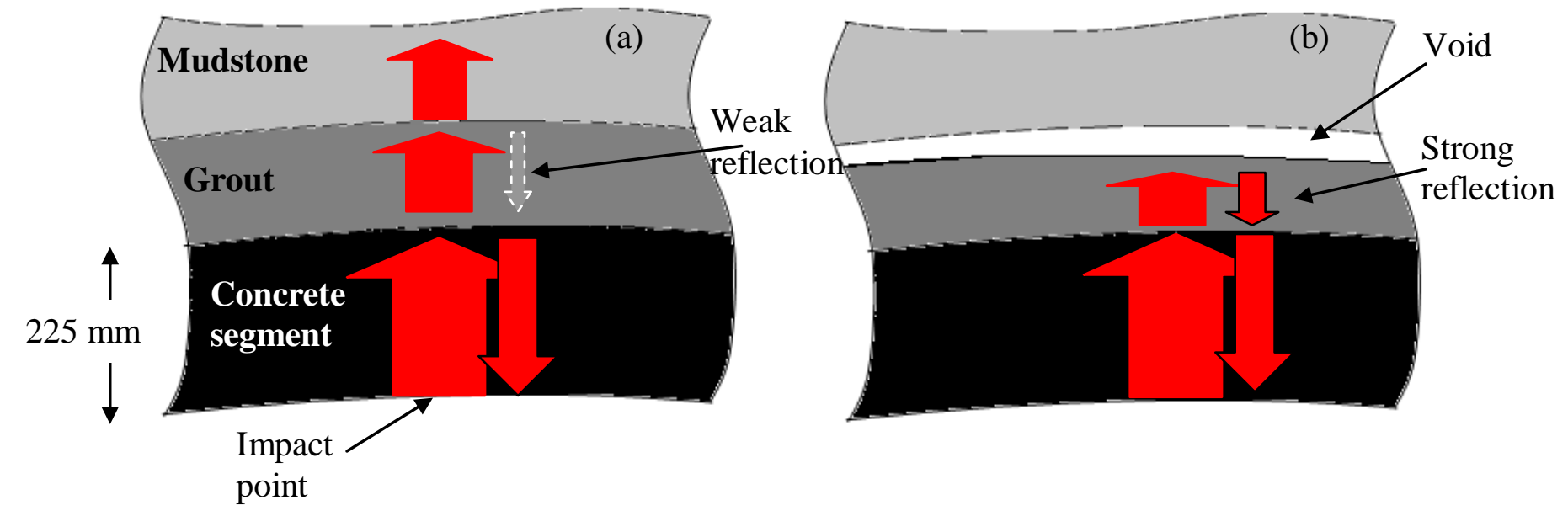

Fig. 3 Reflections according to the internal geometry (a) fully filled case, (b) partially filled case. 

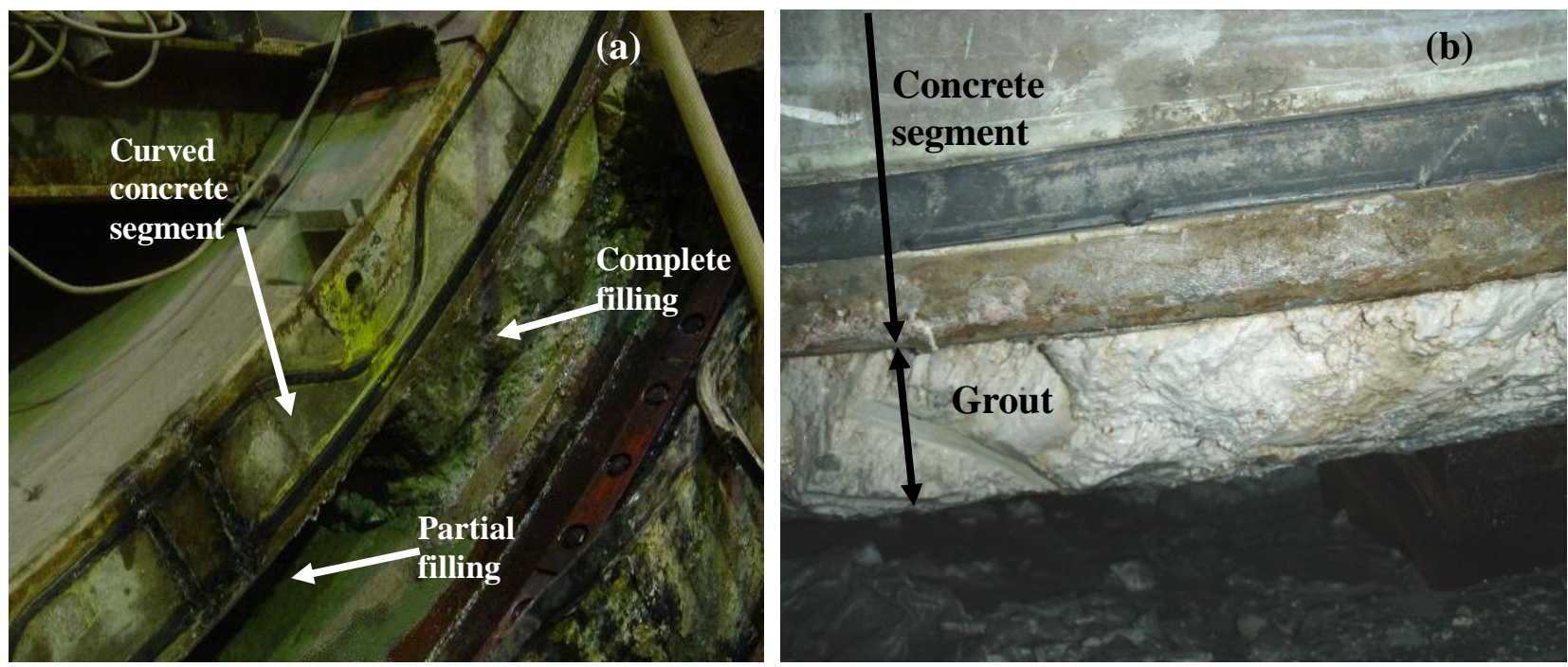

Fig. 4 (a) Different filling cases simulated at the entrance of the tunnel, (b) detail of partial filling case. 

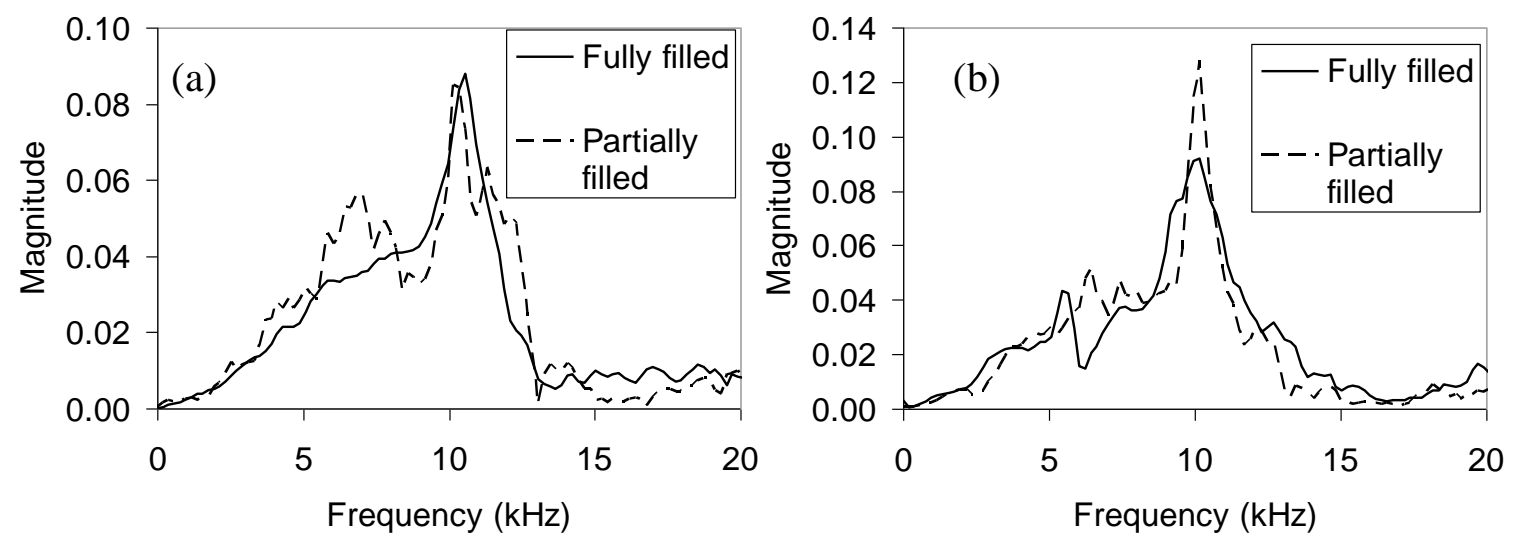

Fig. 5 FFT of different filling cases. (a) distinguishable cases, (b) not distinguishable cases. 


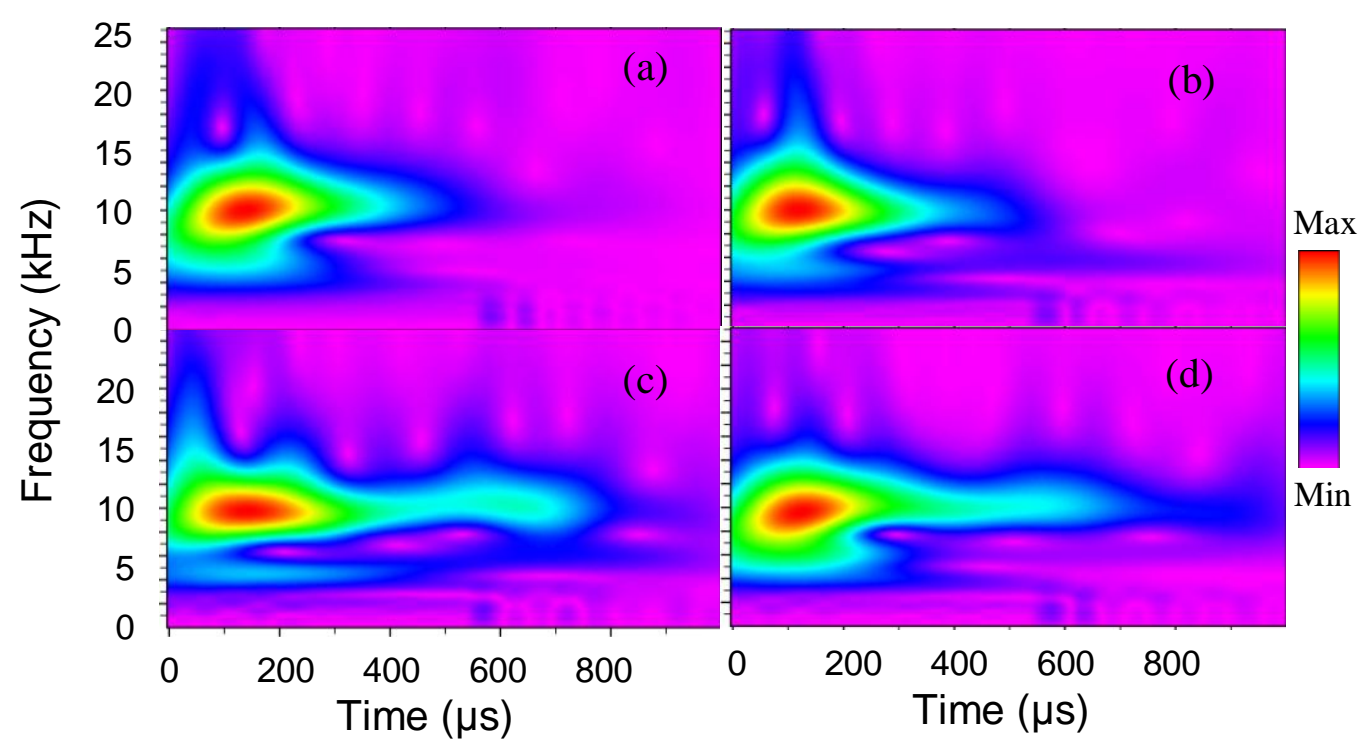

Fig. 6 Wavelet transforms of responses from: (a) and (b) fully filled, (c) and (d) partially filled position. 

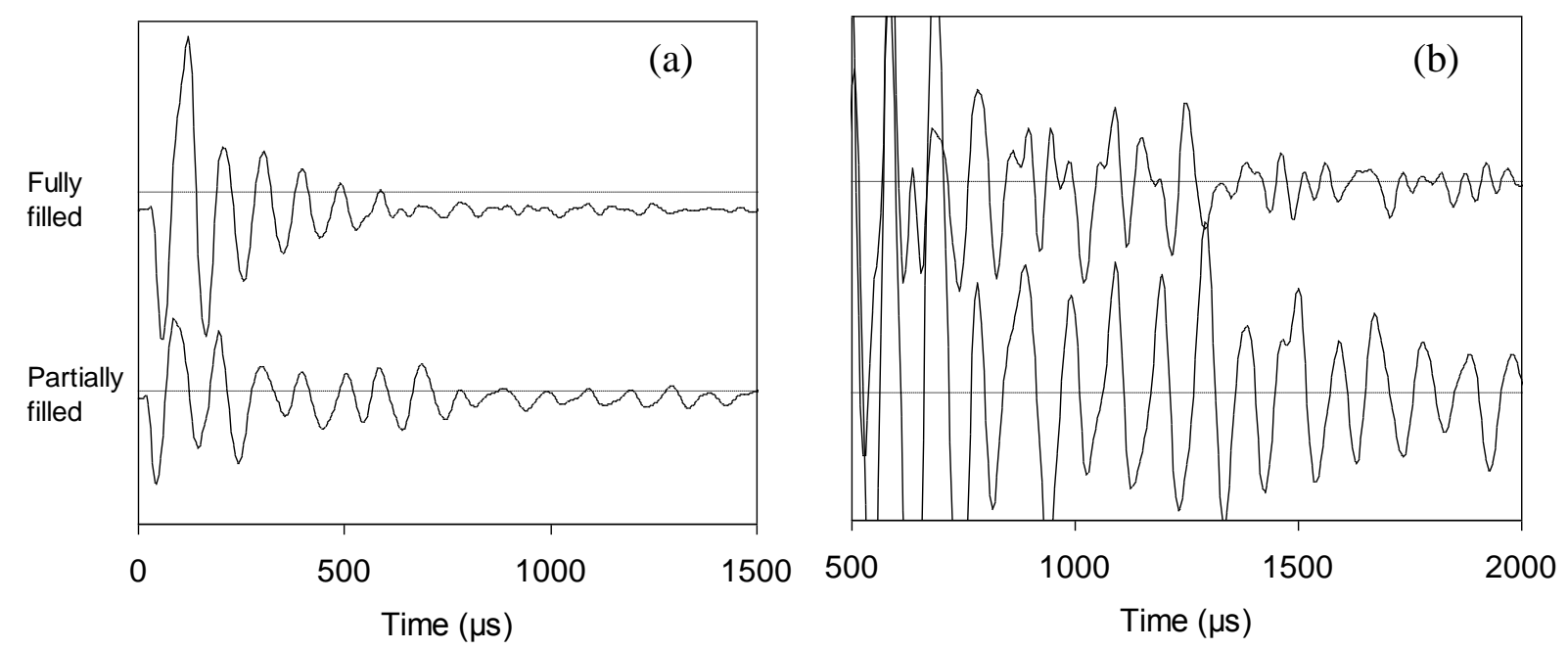

Fig. 7 (a) Waveforms from different filling cases with threshold $10 \%$ of the maximum, (b) the waveforms magnified after $500 \mu$ s, with threshold $0.1 \%$. 

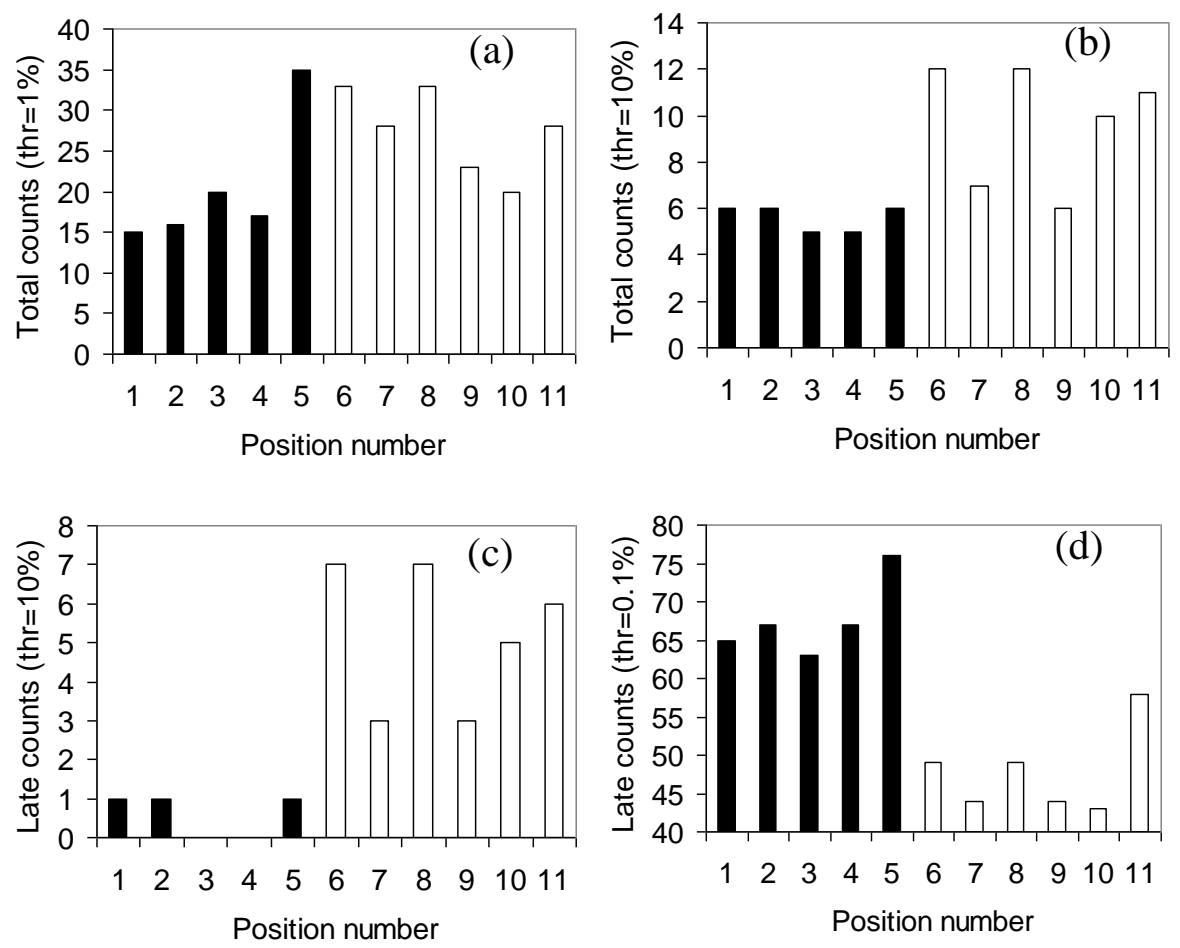

Fig. 8 Number of (a) counts for threshold $1 \%$ of maximum voltage, (b) counts for threshold $10 \%$ of maximum voltage, (c) counts after $500 \mu$ s for threshold $10 \%$, (d) counts after $500 \mu \mathrm{s}$ for threshold $0.1 \%$ (positions 1-5 correspond to full filling and 6-11 to partial filling). 


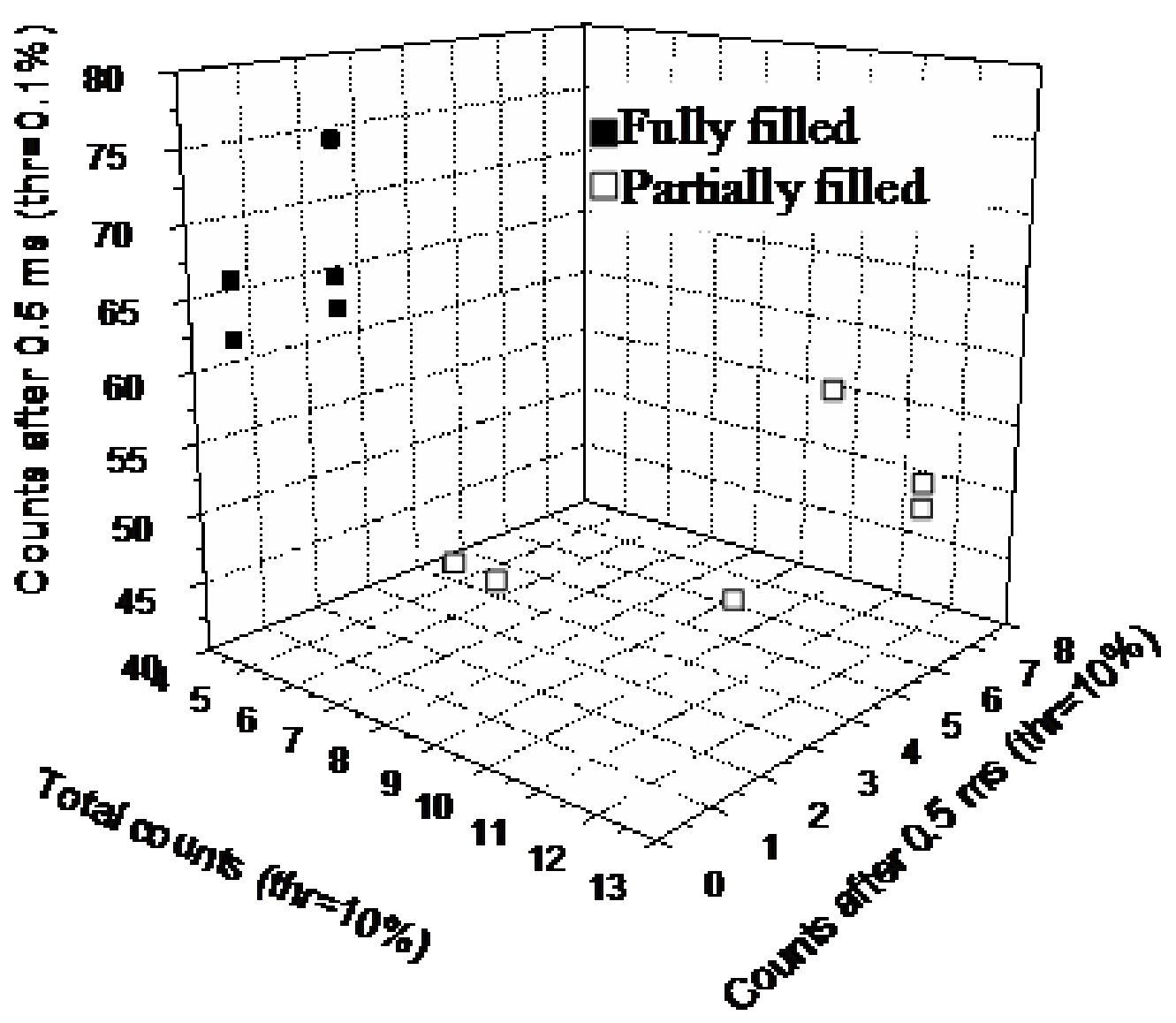

Fig. 9 Three dimensional representation of different clusters. 

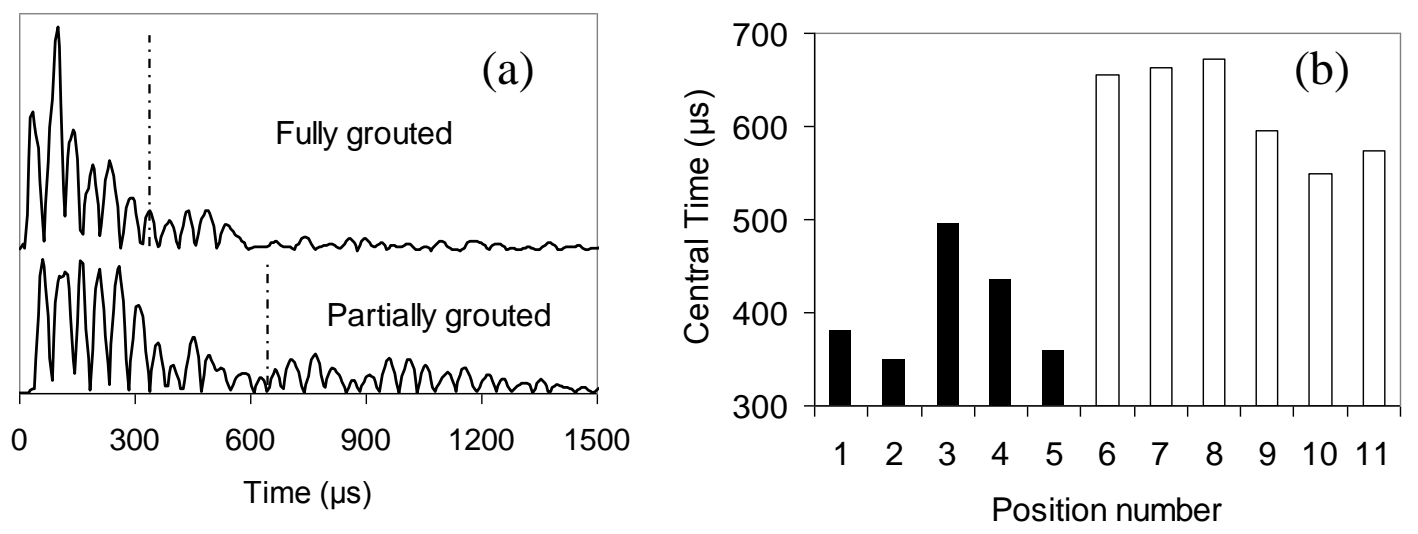

Fig. 10 (a) Rectified waveforms for different grouting cases (the dash-dot line corresponds to the calculated centroid, (b) central waveform time for different testing points (positions 1-5 correspond to full filling and 6-11 to partial filling). 

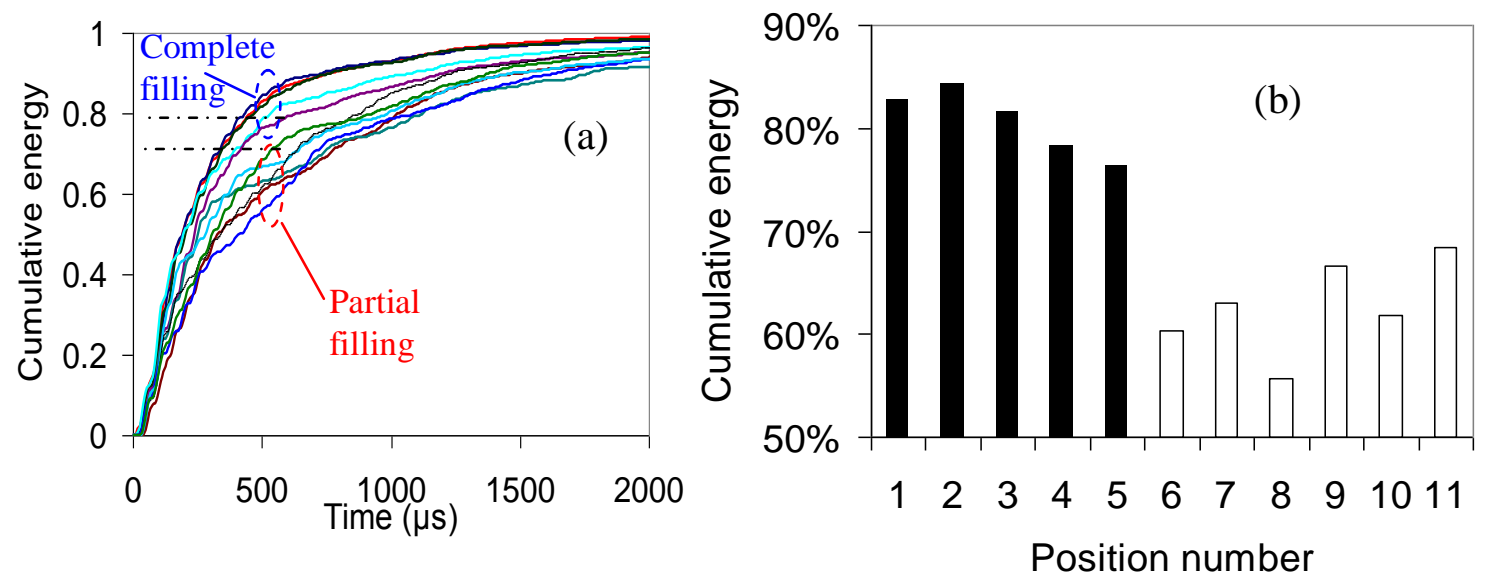

Fig. 11 (a) Normalized cumulative energy for different testing points, (b) Normalized cumulative energy at the time of $500 \mu$ s (positions 1-5 correspond to full filling and 6-11 to partial filling). 